\title{
A systematic review of the prevalence and determinants of nonadherence to phosphate binding medication in patients with end-stage renal disease
} Christina Karamanidou ${ }^{1}$, Jane Clatworthy ${ }^{1}$, John Weinman ${ }^{2}$ and Rob Horne*1

Address: ${ }^{1}$ Centre for Behavioural Medicine, The School of Pharmacy, University of London, Mezzanine Floor, BMA House, Tavistock Square, London WC1H 9JP, UK and ${ }^{2}$ Health Psychology Section, Institute of Psychiatry, University of London, 5th floor Thomas Guy House, London Bridge, London SE1 9RT, UK

Email: Christina Karamanidou - psy77ck@yahoo.com; Jane Clatworthy - jane.clatworthy@pharmacy.ac.uk; John Weinman - john.weinman@kcl.ac.uk; Rob Horne* - rob.horne@pharmacy.ac.uk

* Corresponding author

Published: 31 January 2008

BMC Nephrology 2008, 9:2 doi:10.1/86/147|-2369-9-2
Received: 28 August 2007

Accepted: 31 January 2008

This article is available from: http://www.biomedcentral.com/I47I-2369/9/2

(c) 2008 Karamanidou et al; licensee BioMed Central Ltd.

This is an Open Access article distributed under the terms of the Creative Commons Attribution License (http://creativecommons.org/licenses/by/2.0), which permits unrestricted use, distribution, and reproduction in any medium, provided the original work is properly cited.

\begin{abstract}
Background: Cardiovascular events are the leading cause of death in end stage renal disease (ESRD). Adherence to phosphate binding medication plays a vital role in reducing serum phosphorus and associated cardiovascular risk. This poses a challenge for patients as the regimen is often complex and there may be no noticeable impact of adherence on symptoms. There is a need to establish the level of nonadherence to phosphate binding medication in renal dialysis patients and identify the factors associated with it.

Methods: The online databases PsycINFO, Medline, Embase and CINAHL were searched for quantitative studies exploring predictors of nonadherence to phosphate binding medication in ESRD. Rates and predictors of nonadherence were extracted from the papers.

Results: Thirty four studies met the inclusion criteria. There was wide variation in reported rates of non-adherence (22-74\% patients nonadherent, mean $51 \%$ ). This can be partially attributed to differences in the way adherence has been defined and measured. Demographic and clinical predictors of nonadherence were most frequently assessed but only younger age was consistently associated with nonadherence. In contrast psychosocial variables (e.g. patients' beliefs about medication, social support, personality characteristics) were less frequently assessed but were more likely to be associated with nonadherence.
\end{abstract}

Conclusion: Nonadherence to phosphate binding medication appears to be prevalent in ESRD. Several potentially modifiable psychosocial factors were identified as predictors of nonadherence. There is a need for further, high-quality research to explore these factors in more detail, with the aim of informing the design of an intervention to facilitate adherence. 


\section{Background}

Cardiovascular events constitute the leading cause of death in dialysis patients, accounting for nearly half of all deaths [1]. The increased incidence of cardiac disease in patients with end-stage renal disease (ESRD) has been associated with hyperphosphatemia and more specifically an elevated Calcium $\times$ Phosphate product [2], making phosphate control an important goal of treatment. Three strategies help to control serum phosphate in ESRD: dialysis, diet restrictions and phosphate binding medication.

Adherence to phosphate binding medication may be a particular challenge for dialysis patients, due to complex treatment regimens that may have no noticeable effect on symptoms. Many patients on dialysis are nonadherent with phosphate binding medication [3] but the extent of the problem and the reasons for it are poorly understood.

There is a current emphasis in the literature on the importance of facilitating adherence to medication in chronic illnesses [4-6]. In order to develop a theory-based intervention to optimise adherence to phosphate binding medication, there is a need to first understand the factors associated with nonadherence that could be addressed. Previous reviews of predictors of adherence in ESRD have tended to focus on other aspects of the regime (e.g. diet and fluid restrictions, dialysis attendance) [7-10] and have not used comprehensive systematic search strategies $[7,8,11]$. This is the first paper to systematically search and review the literature specifically relating to adherence to phosphate binding medication.

The aims of this review were to assess the prevalence of nonadherence to phosphate binding medication in patients with ESRD and to identify factors associated with low adherence.

\section{Methods}

Articles were identified for review through the search of online databases PsycINFO (1967-2006), Medline (1950-2006), Embase (1980-2006) and CINAHL (1982-2006). Papers with abstracts containing a combination of three of the terms shown in Table 1 (one from each column) were selected.

Table I: Terms used in database searches (\$ indicates truncation)

\begin{tabular}{lll}
\hline Haemodialysis & Adheren\$ & Medication $\$$ \\
Hemodialysis & Nonadheren\$ & Phosphate binder $\$$ \\
Uremic & Complian\$ & Serum phosphate \\
Dialysis & Noncomplian $\$$ & Serum phosphorus \\
Renal disease & & Regimen $\$$
\end{tabular}

Note. The search term 'dialysis' retrieved studies involving participants receiving peritoneal dialysis, therefore the papers reviewed included participants receiving both peritoneal dialysis and hemodialyis.
The database search resulted in the identification of 481 papers. Three additional papers were identified through a reference list search. Each paper was evaluated by two independent reviewers. Papers were retained if they contained quantitative studies exploring predictors of nonadherence to $\mathrm{PB}$ medication in ESRD, were published in English and were available from the British Library. Qualitative studies were excluded because this review aimed to quantify the number of studies reporting a statistically significant relationship versus the number of studies finding no significant relationship between each possible predictor of nonadherence and nonadherence. Papers were also excluded if they focused on paediatric adherence (patients under 18 years old), were review articles, intervention studies or case studies, or contained secondary analyses on data already included in this review.

The two reviewers extracted information on the rates of nonadherence reported and the predictors of nonadherence explored in each paper. A list of all the variables that had been investigated in relation to nonadherence was compiled. These possible predictors of nonadherence were divided into three categories: demographic, clinical and psychosocial. The number of studies reporting a significant relationship $(\mathrm{p}<.05)$ between each variable and nonadherence and the number of studies reporting no significant relationship ( $\mathrm{p}>.05$ ) between each variable and nonadherence were recorded.

\section{Results and discussion}

Thirty four studies fulfilled the inclusion criteria. Key information extracted from these papers is presented in Table 2.

\section{Prevalence of nonadherence}

Only 13 studies reported rates of nonadherence to phosphate binding medication. Estimates of the percentage of nonadherent participants ranged from 22-74\% (mean $51 \%$ ). This variation can in part be attributed to differences in the way in which nonadherence was measured and defined, for example, the mean number of people classified as nonadherent when assessed through serum phosphorus levels was 58\%, compared to 31\% when assessed using self report measures.

These measurement issues are discussed in more detail under limitations of the studies reviewed.

\section{Predictors of nonadherence \\ Demographic variables}

The most frequently assessed demographic predictors of phosphate binder adherence were age ( 24 studies), gender (22 studies), educational level (21 studies), marital status (11 studies), ethnicity ( 8 studies), income (6 studies) and employment status (3 studies). As shown in Figure 1, few 
Table 2: Details of the studies included in the review

\begin{tabular}{|c|c|c|c|c|c|c|c|c|c|c|c|}
\hline \multirow[t]{2}{*}{ Study } & \multirow{2}{*}{$\begin{array}{l}\mathbf{N} \\
\text { Dialysis } \\
\text { type }\end{array}$} & \multirow{2}{*}{$\begin{array}{l}\text { Mean } \\
\text { age } \\
\text { (years) }\end{array}$} & \multirow{2}{*}{$\begin{array}{l}\text { Mean } \\
\text { time on } \\
\text { dialysis } \\
\text { (months) }\end{array}$} & \multirow{2}{*}{$\begin{array}{l}\text { Gender } \\
\% \text { male }\end{array}$} & \multirow{2}{*}{$\begin{array}{l}\text { Main } \\
\text { statistical } \\
\text { analysis }\end{array}$} & \multicolumn{3}{|c|}{ Predictors of adherence } & \multirow{2}{*}{$\begin{array}{l}\text { Adherence } \\
\text { assessment }\end{array}$} & \multirow{2}{*}{$\begin{array}{l}\text { Non- } \\
\text { adherence } \\
\text { definition }\end{array}$} & \multirow{2}{*}{$\begin{array}{l}\% \text { Non- } \\
\text { adherent }\end{array}$} \\
\hline & & & & & & Demographic & Clinical & Psychosocial & & & \\
\hline $\begin{array}{l}\text { Bame et al, } 1993 \\
\text { U.S. [12] }\end{array}$ & $\begin{array}{l}1230 \\
\mathrm{HD}\end{array}$ & 57 & Not stated & $47 \%$ & $\begin{array}{l}\text { Multiple } \\
\text { logistic } \\
\text { regression }\end{array}$ & $\begin{array}{l}\text { Age*, income*, } \\
\text { gender, } \\
\text { ethnicity, } \\
\text { marital status, } \\
\text { education }\end{array}$ & $\begin{array}{l}\text { Not } \\
\text { assessed }\end{array}$ & Not assessed & $\begin{array}{l}\text { Serum } \\
\text { phosphorus }\end{array}$ & $>6 \mathrm{mg} / \mathrm{dl}$ & $50 \%$ \\
\hline $\begin{array}{l}\text { Betts \& Crotty, } \\
\text { I } 988 \text { U.S.[42] }\end{array}$ & $46 \mathrm{HD}$ & $\begin{array}{l}\text { Not } \\
\text { stated }\end{array}$ & Not stated & $33 \%$ & Correlations & Age, education & $\begin{array}{l}\text { Time on } \\
\text { dialysis }\end{array}$ & Response to illness & $\begin{array}{l}\text { Serum } \\
\text { phosphorus }\end{array}$ & $>5 \mathrm{mg} / \mathrm{dl}$ & $74 \%$ \\
\hline $\begin{array}{l}\text { Blanchard et al, } \\
\text { I } 990 \text { U.S.[38] }\end{array}$ & $\begin{array}{l}40 \mathrm{HD} \\
40 \mathrm{PD}\end{array}$ & 50 & Not stated & $48 \%$ & Correlations & Gender & $\begin{array}{l}\text { Time on } \\
\text { dialysis }\end{array}$ & Not assessed & Self report & $\begin{array}{l}\text { Reporting } \\
\text { ever missing a } \\
\text { dose }\end{array}$ & $28 \%$ \\
\hline $\begin{array}{l}\text { Boyer et al, } 1990 \\
\text { U.S.[13] }\end{array}$ & $60 \mathrm{HD}$ & $\begin{array}{l}\text { Not } \\
\text { stated }\end{array}$ & Not stated & $71 \%$ & $\begin{array}{l}\text { Correlations } \\
\text {, multiple } \\
\text { regression }\end{array}$ & $\begin{array}{l}\text { Age*, marital } \\
\text { status*, gender, } \\
\text { ethnicity, } \\
\text { income, } \\
\text { education }\end{array}$ & $\begin{array}{l}\text { Time on } \\
\text { dialysis }\end{array}$ & Social support* & $\begin{array}{l}\text { Serum } \\
\text { phosphorus }\end{array}$ & $\begin{array}{l}\text { Not } \\
\text { dichotomised }\end{array}$ & Not stated \\
\hline $\begin{array}{l}\text { Christensen et al, } \\
\text { 1994 U.S.[14] }\end{array}$ & $\begin{array}{l}52 \mathrm{HD} \\
34 \mathrm{PD}\end{array}$ & 49 & 73 & $53 \%$ & $\begin{array}{l}\text { Hierarchical } \\
\text { regression }\end{array}$ & $\begin{array}{l}\text { Age**, gender, } \\
\text { marital status, } \\
\text { education }\end{array}$ & $\begin{array}{l}\text { Diabetic } \\
\text { status, time } \\
\text { on dialysis, } \\
\text { transplant } \\
\text { history, type } \\
\text { of dialysis }\end{array}$ & $\begin{array}{l}\text { Information } \\
\text { vigilance, active } \\
\text { coping }\end{array}$ & $\begin{array}{l}\text { Serum } \\
\text { phosphorus }\end{array}$ & $\begin{array}{l}\text { Not } \\
\text { dichotomised }\end{array}$ & Not stated \\
\hline $\begin{array}{l}\text { Christensen et al, } \\
1995 \text { U.S.[15] }\end{array}$ & $\begin{array}{l}72 \mathrm{HD} \\
\text { or PD }\end{array}$ & 46 & 73 & $54 \%$ & $\begin{array}{l}\text { Correlations } \\
\text {, stepwise } \\
\text { regression }\end{array}$ & Age*, education & $\begin{array}{l}\text { Time on } \\
\text { dialysis, } \\
\text { transplant } \\
\text { history }\end{array}$ & $\begin{array}{l}\text { Neuroticism, } \\
\text { extraversion, } \\
\text { openness to } \\
\text { experience, } \\
\text { agreeableness, } \\
\text { conscientiousness* }\end{array}$ & $\begin{array}{l}\text { Serum } \\
\text { phosphorus }\end{array}$ & $\begin{array}{l}\text { Not } \\
\text { dichotomised }\end{array}$ & Not stated \\
\hline $\begin{array}{l}\text { Christensen et al, } \\
\text { 1996a U.S.[44] }\end{array}$ & $52 \mathrm{HD}$ & 59 & 62 & $59 \%$ & Regression & $\begin{array}{l}\text { Age, education, } \\
\text { gender }\end{array}$ & $\begin{array}{l}\text { Diabetic } \\
\text { status, time } \\
\text { on dialysis }\end{array}$ & $\begin{array}{l}\text { Neuroticism, } \\
\text { private body } \\
\text { consciousness, } \\
\text { illness related } \\
\text { physical impairment }\end{array}$ & $\begin{array}{l}\text { Serum } \\
\text { phosphorus }\end{array}$ & $\begin{array}{l}\text { Not } \\
\text { dichotomised }\end{array}$ & Not stated \\
\hline $\begin{array}{l}\text { Christensen et al, } \\
\text { I996b U.S. [16] }\end{array}$ & $\begin{array}{l}67 \mathrm{HD} \\
14 \mathrm{PD}\end{array}$ & 55 & 70 & $49 \%$ & Regression & $\begin{array}{l}\text { Age **, } \\
\text { education, } \\
\text { gender }\end{array}$ & $\begin{array}{l}\text { Diabetic } \\
\text { status*, type } \\
\text { of dialysis, } \\
\text { time on } \\
\text { dialysis }\end{array}$ & $\begin{array}{l}\text { Perceived health } \\
\text { competence*, } \\
\text { health locus of } \\
\text { control }\end{array}$ & $\begin{array}{l}\text { Serum } \\
\text { phosphorus }\end{array}$ & $\begin{array}{l}\text { Not } \\
\text { dichotomised }\end{array}$ & Not stated \\
\hline $\begin{array}{l}\text { Christensen et al, } \\
\text { 1997a U.S.[33] }\end{array}$ & $5 I \mathrm{HD}$ & 57 & 51 & $59 \%$ & $\begin{array}{l}\text { Correlation, } \\
\text { hierarchical } \\
\text { regression }\end{array}$ & $\begin{array}{l}\text { Age, education, } \\
\text { gender }\end{array}$ & $\begin{array}{l}\text { Diabetic } \\
\text { status, time } \\
\text { on dialysis }\end{array}$ & $\begin{array}{l}\text { Monitoring } \\
\text { attentional style, } \\
\text { trait anxiety, } \\
\text { internal health locus } \\
\text { of control, control } \\
\text { appraisal, avoidant } \\
\text { coping }\end{array}$ & $\begin{array}{l}\text { Serum } \\
\text { phosphorus }\end{array}$ & $\begin{array}{l}\text { Not } \\
\text { dichotomised }\end{array}$ & Not stated \\
\hline $\begin{array}{l}\text { Christensen et al, } \\
\text { 1997b U.S.[43] }\end{array}$ & $48 \mathrm{HD}$ & 56 & 65 & $54 \%$ & $\begin{array}{l}\text { Correlations } \\
\text {, hierarchical } \\
\text { regression }\end{array}$ & $\begin{array}{l}\text { Age, education, } \\
\text { gender }\end{array}$ & $\begin{array}{l}\text { Diabetic } \\
\text { status, time } \\
\text { on dialysis }\end{array}$ & $\begin{array}{l}\text { Cynical hostility*, } \\
\text { health locus of } \\
\text { control }\end{array}$ & $\begin{array}{l}\text { Serum } \\
\text { phosphorus }\end{array}$ & $\begin{array}{l}\text { Not } \\
\text { dichotomised }\end{array}$ & Not stated \\
\hline $\begin{array}{l}\text { Cummings et al, } \\
\text { I } 982 \text { U.S.[22] }\end{array}$ & $116 \mathrm{HD}$ & 55 & 29 & $54 \%$ & $\begin{array}{l}\text { Correlations } \\
\text {, regression }\end{array}$ & $\begin{array}{l}\text { Age*, gender, } \\
\text { income, } \\
\text { education }\end{array}$ & $\begin{array}{l}\text { Time on } \\
\text { dialysis, } \\
\text { transplant } \\
\text { history, } \\
\text { regimen } \\
\text { complexity* }\end{array}$ & $\begin{array}{l}\text { Susceptibility, } \\
\text { severity, benefits*+, } \\
\text { barriers', } \\
\text { knowledge of } \\
\text { purpose of } \\
\text { regimen*, social } \\
\text { support (family and } \\
\text { friends), support } \\
\text { from medical staff } \\
\text { family problems }^{+} \text {, }\end{array}$ & $\begin{array}{l}\text { Serum } \\
\text { phosphorus } \\
\text { Self report }\end{array}$ & $>5.5 \mathrm{mg} / \mathrm{dl}$ & $\begin{array}{l}70 \% \text { (based } \\
\text { on serum } \\
\text { phosphorus) }\end{array}$ \\
\hline $\begin{array}{l}\text { Curtin et al, } 1999 \\
\text { U.S.[49] }\end{array}$ & I35 HD & 63 & Not stated & $47 \%$ & Chi-square & $\begin{array}{l}\text { Ethnicity } \\
\text { age, gender, } \\
\text { employment } \\
\text { status, } \\
\text { education }\end{array}$ & $\begin{array}{l}\text { Cause of } \\
\text { renal failure, } \\
\text { no. } \\
\text { comorbiditi } \\
\text { es, time on } \\
\text { dialysis }\end{array}$ & Not assessed & $\begin{array}{l}\text { Electronic } \\
\text { monitoring } \\
\text { (used in } \\
\text { analysis) } \\
\text { Pill count } \\
\text { Self report }\end{array}$ & $\begin{array}{l}\text { Overdosing/ } \\
\text { underdosing/ } \\
\text { missing more } \\
\text { than } 20 \% \\
\text { prescribed } \\
\text { doses }\end{array}$ & $\begin{array}{l}73 \% \text { (based } \\
\text { on } \\
\text { electronic } \\
\text { monitoring) }\end{array}$ \\
\hline $\begin{array}{l}\text { Eitel et al, } 1998 \\
\text { U.S.[27] }\end{array}$ & $\begin{array}{l}40 \mathrm{HD} \\
45 \mathrm{PD}\end{array}$ & 55 & 40 & $61 \%$ & Correlations & Not assessed & $\begin{array}{l}\text { Not } \\
\text { assessed }\end{array}$ & $\begin{array}{l}\text { Efficacy } \\
\text { expectations**, } \\
\text { effort attributions, } \\
\text { self control }\end{array}$ & $\begin{array}{l}\text { Serum } \\
\text { phosphorus } \\
\text { (used in } \\
\text { analysis) } \\
\text { Self report } \\
\text { Staff ratings }\end{array}$ & $\begin{array}{l}\text { Not } \\
\text { dichotomised }\end{array}$ & Not stated \\
\hline $\begin{array}{l}\text { Gago et al, } 2000 \\
\text { Spain[35] }\end{array}$ & $121 \mathrm{HD}$ & 63 & 37 & $56 \%$ & T-tests & $\begin{array}{l}\text { Gender, age, } \\
\text { living } \\
\text { arrangements }\end{array}$ & $\begin{array}{l}\text { Cause of } \\
\text { ESRD, time } \\
\text { on dialysis }\end{array}$ & Knowledge & Not clear & Not clear & $46 \%$ \\
\hline $\begin{array}{l}\text { Hilbert, } 1985 \\
\text { U.S.[29] }\end{array}$ & $26 \mathrm{HD}$ & 47 & 54 & $35 \%$ & $\begin{array}{l}\text { Correlations } \\
\text {, ANOVA }\end{array}$ & $\begin{array}{l}\text { Age, income, } \\
\text { education, } \\
\text { social class, } \\
\text { religion, gender, } \\
\text { significant other }\end{array}$ & $\begin{array}{l}\text { No. times } \\
\text { hospitalised, } \\
\text { time on } \\
\text { dialysis }^{+}\end{array}$ & $\begin{array}{l}\text { Directive guidance } \\
\text { social support } \\
\text { affection social } \\
\text { support }\end{array}$ & $\begin{array}{l}\text { Composite } \\
\text { self report } \\
\text { scale- } \\
\text { adherence to } \\
\text { medication, } \\
\text { fluid and diet } \\
\text { (used in } \\
\text { analysis) } \\
\text { Serum } \\
\text { phosphorus }\end{array}$ & $\begin{array}{l}\text { Not } \\
\text { dichotomised }\end{array}$ & Not stated \\
\hline
\end{tabular}


Table 2: Details of the studies included in the review (Continued)

\begin{tabular}{|c|c|c|c|c|c|c|c|c|c|c|c|}
\hline $\begin{array}{l}\text { Horne et al, 200I } \\
\text { U.K.[I7] }\end{array}$ & $47 \mathrm{HD}$ & 49 & 53 & $49 \%$ & Correlations & $\begin{array}{l}\mathrm{Age}^{+} \text {, gender, } \\
\text { education }\end{array}$ & $\begin{array}{l}\text { Duration of } \\
\text { ESRD, time } \\
\text { on dialysis, } \\
\text { no. } \\
\text { prescribed } \\
\text { medicines }\end{array}$ & $\begin{array}{l}\text { Beliefs about } \\
\text { medication } \\
\text { (concerns }{ }^{++}, \\
\text {perceived need, } \\
\text { harm, overuse) }\end{array}$ & Self report & $\begin{array}{l}\text { Those who } \\
\text { reported } \\
\text { sometimes, } \\
\text { often or very } \\
\text { often } \\
\text { deliberately } \\
\text { missing a dose } \\
\text { of their } \\
\text { medication. }\end{array}$ & $26 \%$ \\
\hline $\begin{array}{l}\text { Leggat et al, } 1998 \\
\text { U.S.[18] }\end{array}$ & $\begin{array}{l}6251 \\
H D\end{array}$ & 58 & 54 & $50 \%$ & $\begin{array}{l}\text { Logistic } \\
\text { regression }\end{array}$ & $\begin{array}{l}\text { Age****, } \\
\text { ethnicity*, } \\
\text { smoker*, } \\
\text { gender, } \\
\text { education, living } \\
\text { arrangements }\end{array}$ & $\begin{array}{l}\text { Time on } \\
\text { dialysis, } \\
\text { diabetic } \\
\text { status, } \\
\text { transplant } \\
\text { history }\end{array}$ & Not assessed & $\begin{array}{l}\text { Serum } \\
\text { phosphorus }\end{array}$ & $>7.5$ mg/dl & $22 \%$ \\
\hline $\begin{array}{l}\text { Lin \& Liang, } 1997 \\
\text { China[39] }\end{array}$ & $86 \mathrm{HD}$ & 55 & 42 & $45 \%$ & Correlations & Not assessed & $\begin{array}{l}\text { Not } \\
\text { assessed }\end{array}$ & $\begin{array}{l}\text { Health locus of } \\
\text { control }^{+++} * * *\end{array}$ & $\begin{array}{l}3 \text { composite } \\
\text { measures: } \\
\text { Lab reports } \\
\text { (including } \\
\text { serum } \\
\text { phosphorus) } \\
\text { Self report - } \\
\text { fluid, diet and } \\
\text { medication } \\
\text { adherence } \\
\text { Nurses' } \\
\text { assessment - } \\
\text { fluid, diet and } \\
\text { medication } \\
\text { adherence }\end{array}$ & $>4.59 \mathrm{mg} / \mathrm{dl}$ & $\begin{array}{l}61 \% \text { (based } \\
\text { on serum } \\
\text { phosphorus) }\end{array}$ \\
\hline $\begin{array}{l}\text { Moran et al, } 1997 \\
\text { U.S.[45] }\end{array}$ & $56 \mathrm{HD}$ & 57 & 46 & $64 \%$ & Regression & $\begin{array}{l}\text { Age, gender, } \\
\text { education }\end{array}$ & $\begin{array}{l}\text { Time on } \\
\text { dialysis, } \\
\text { diabetic } \\
\text { status, } \\
\text { transplant } \\
\text { history*** }\end{array}$ & $\begin{array}{l}\text { Social support, } \\
\text { conscientiousness }\end{array}$ & $\begin{array}{l}\text { Serum } \\
\text { phosphorus }\end{array}$ & $\begin{array}{l}\text { Not } \\
\text { dichotomised }\end{array}$ & Not stated \\
\hline $\begin{array}{l}\text { Morduchowicz et } \\
\text { al, I993 } \\
\text { Israel[46] }\end{array}$ & $50 \mathrm{HD}$ & 56 & 66 & $60 \%$ & $\begin{array}{l}\text { Multivariate } \\
\text { and stepwise } \\
\text { regression }\end{array}$ & $\begin{array}{l}\text { Education } * * * \text {, } \\
\text { ethnicity*, } \\
\text { gender, age, } \\
\text { place of birth, } \\
\text { religious } \\
\text { observance, } \\
\text { marital status, } \\
\text { no. children, } \\
\text { whether } \\
\text { accompanied to } \\
\text { session, } \\
\text { economic } \\
\text { status, living } \\
\text { arrangements }\end{array}$ & $\begin{array}{l}\text { Previous PD } \\
\text { dialysis, time } \\
\text { on dialysis }\end{array}$ & Not assessed & $\begin{array}{l}\text { Serum } \\
\text { phosphorus }\end{array}$ & $\begin{array}{l}\text { Not } \\
\text { dichotomised }\end{array}$ & Not stated \\
\hline $\begin{array}{l}\text { O'Brien, } 1980 \\
\text { U.S.[28] }\end{array}$ & $63 \mathrm{HD}$ & $\begin{array}{l}\text { Not } \\
\text { stated }\end{array}$ & Not stated & $54 \%$ & $\begin{array}{l}\text { ANOVA, } \\
\text { correlations, } \\
\text { regression }\end{array}$ & $\begin{array}{l}\text { Age, gender, } \\
\text { marital status }{ }^{++} \text {, } \\
\text { ethnicity, } \\
\text { education, } \\
\text { occupation, } \\
\text { type of } \\
\text { household }\end{array}$ & $\begin{array}{l}\text { Time on } \\
\text { dialysis }\end{array}$ & $\begin{array}{l}\text { Significant others' } \\
\text { expectations } \\
\text { regarding }^{\text {adherence }} \\
\text { ad+ }^{++}\end{array}$ & $\begin{array}{l}\text { Composite } \\
\text { self report } \\
\text { scale- } \\
\text { dialysis } \\
\text { attendance, } \\
\text { diet, fluid and } \\
\text { medication }\end{array}$ & Not stated & Not stated \\
\hline $\begin{array}{l}\text { Reiss et al, } 1986 \\
\text { U.S.[47] }\end{array}$ & $23 \mathrm{HD}$ & 48 & 8 & $57 \%$ & Correlations & $\begin{array}{l}\text { Family income, } \\
\text { marital status, } \\
\text { years married, } \\
\text { family size, } \\
\text { education }\end{array}$ & $\begin{array}{l}\text { Not } \\
\text { assessed }\end{array}$ & $\begin{array}{l}\text { Problem solving } \\
\text { (coordination and } \\
\text { closure), family } \\
\text { intelligence }\end{array}$ & $\begin{array}{l}\text { Serum } \\
\text { phosphorus }\end{array}$ & $\begin{array}{l}\text { Not } \\
\text { dichotomised }\end{array}$ & Not stated \\
\hline $\begin{array}{l}\text { Schlebusch \& } \\
\text { Levin, I982 } \\
\text { South Africa[34] }\end{array}$ & $\begin{array}{l}25 \mathrm{HD} \\
\text { or PD }\end{array}$ & 38 & Not stated & $48 \%$ & $\begin{array}{l}\text { Mann- } \\
\text { Whitney } \\
\text { test }\end{array}$ & Not assessed & $\begin{array}{l}\text { Organicity } \\
\text { (cortical } \\
\text { dysfunction) }\end{array}$ & $\begin{array}{l}\text { Intelligence, } \\
\text { personality\$\$ }\end{array}$ & $\begin{array}{l}\text { Composite } \\
\text { staff rating - } \\
\text { including } \\
\text { adherence to } \\
\text { medication } \\
\text { and diet }\end{array}$ & Not stated & $44 \%$ \\
\hline $\begin{array}{l}\text { Schneider, } 1992 \\
\text { U.S.[19] }\end{array}$ & I37 HD & 51 & 26 & $54 \%$ & $\begin{array}{l}\text { Multiple } \\
\text { regression }\end{array}$ & $\begin{array}{l}\text { Age****, gender, } \\
\text { ethnicity, } \\
\text { education }\end{array}$ & $\begin{array}{l}\text { Time on } \\
\text { dialysis, } \\
\text { frequency of } \\
\text { physician } \\
\text { contact }\end{array}$ & $\begin{array}{l}\text { Health locus of } \\
\text { control**** }\end{array}$ & $\begin{array}{l}\text { Serum } \\
\text { phosphorus }\end{array}$ & $\begin{array}{l}\text { Not } \\
\text { dichotomized }\end{array}$ & Not stated \\
\hline $\begin{array}{l}\text { Sherwood, } 1983 \\
\text { U.S.[30] }\end{array}$ & $55 \mathrm{HD}$ & 46 & 48 & $66 \%$ & Correlations & Not assessed & $\begin{array}{l}\text { Not } \\
\text { assessed }\end{array}$ & $\begin{array}{l}\text { Family } \\
\text { understanding, } \\
\text { family organisation*, } \\
\text { supportive } \\
\text { family**,+++ }\end{array}$ & $\begin{array}{l}\text { Serum } \\
\text { phosphorus } \\
\text { Composite } \\
\text { self-report } \\
\text { measure- } \\
\text { diet, fluid and } \\
\text { medication }\end{array}$ & Not stated & Not stated \\
\hline $\begin{array}{l}\text { Stamatakis et al, } \\
\text { I } 997 \text { U.S.[20] }\end{array}$ & $\begin{array}{l}17 \mathrm{HD} \\
4 \mathrm{PD}\end{array}$ & 53 & Not stated & $48 \%$ & $\begin{array}{l}\text { Anova, chi- } \\
\text { square, } \\
\text { multiple } \\
\text { regression }\end{array}$ & $\begin{array}{l}\text { Age*, gender, } \\
\text { ethnicity } \\
\text { education, } \\
\text { occupation, } \\
\text { marital status }\end{array}$ & $\begin{array}{l}\text { Type of } \\
\text { dialysis, } \\
\text { cause of } \\
\text { ESRD, } \\
\text { transplant } \\
\text { history }\end{array}$ & Knowledge* & $\begin{array}{l}\text { Serum } \\
\text { phosphorus } \\
\text { Self report }\end{array}$ & Not stated & Not stated \\
\hline $\begin{array}{l}\text { Steidl et al, } 1980 \\
\text { U.S.[3 I] }\end{array}$ & $\begin{array}{l}22 \mathrm{HD} \\
\text { I PD }\end{array}$ & 43 & 22 & $57 \%$ & Correlations & Not assessed & $\begin{array}{l}\text { Medical } \\
\text { assessment }\end{array}$ & $\begin{array}{l}\text { Family } \\
\text { functioning } \$ \$ \$\end{array}$ & $\begin{array}{l}\text { Composite } \\
\text { staff } \\
\text { assessment - } \\
\text { dialysis } \\
\text { attendance/ } \\
\text { medication } \\
\text { and diet } \\
\text { adherence }\end{array}$ & Not stated & Not stated \\
\hline $\begin{array}{l}\text { Takaki et al, } 2003 \\
\text { Japan[2I] }\end{array}$ & $484 \mathrm{HD}$ & 60 & 98 & $66 \%$ & $\begin{array}{l}\text { Correlations } \\
\text {, multiple } \\
\text { regression }\end{array}$ & Age****, gender & $\begin{array}{l}\text { Time on } \\
\text { dialysis**** }\end{array}$ & Not assessed & $\begin{array}{l}\text { Serum } \\
\text { phosphorus }\end{array}$ & $\begin{array}{l}\text { Not } \\
\text { dichotomised }\end{array}$ & Not stated \\
\hline
\end{tabular}


Table 2: Details of the studies included in the review (Continued)

\begin{tabular}{|c|c|c|c|c|c|c|c|c|c|c|c|}
\hline $\begin{array}{l}\text { Tomasello et al. } \\
2004 \text { U.S.[3] }\end{array}$ & $\begin{array}{l}129 \mathrm{HD} \\
59 \mathrm{PD}\end{array}$ & 60 & 46 & $\begin{array}{l}\text { Not } \\
\text { stated }\end{array}$ & ANOVA & Age & $\begin{array}{l}\text { Time on } \\
\text { dialysis, } \\
\text { diabetic } \\
\text { status, } \\
\text { tablet } \\
\text { burden* }\end{array}$ & Not assessed & $\begin{array}{l}\text { Self report } \\
\text { Serum } \\
\text { phosphorus }\end{array}$ & $\begin{array}{l}\text { Reporting } \\
\text { taking less } \\
\text { than } 80 \% \\
\text { medication as } \\
\text { prescribed } \\
>5.5 \mathrm{mg} / \mathrm{dl}\end{array}$ & $\begin{array}{l}38 \% \text { (based } \\
\text { on self } \\
\text { report) } 51 \% \\
\text { (based on } \\
\text { serum } \\
\text { phosphorus) }\end{array}$ \\
\hline $\begin{array}{l}\text { Tracy et al, } 1987 \\
\text { U.S.[40] }\end{array}$ & $15 \mathrm{HD}$ & 52 & $\begin{array}{l}0 \text { (starting } \\
\text { dialysis) }\end{array}$ & $67 \%$ & $\begin{array}{l}\text { Correlations } \\
\text {, ANOVA }\end{array}$ & Not assessed & $\begin{array}{l}\text { Not } \\
\text { assessed }\end{array}$ & $\begin{array}{l}\text { Personality*, } \\
\text { depression*, family } \\
\text { environment }\end{array}$ & $\begin{array}{l}\text { Composite } \\
\text { measure - } \\
\text { serum } \\
\text { phosphorus } \\
\text { and } \\
\text { interdialytic } \\
\text { weight }\end{array}$ & Not stated & Not stated \\
\hline $\begin{array}{l}\text { Vives et al, } 1999 \\
\text { Spain[4I] }\end{array}$ & $31 \mathrm{HD}$ & 63 & 35 & $74 \%$ & $\begin{array}{l}\text { Mann } \\
\text { Whitney, } \\
\text { Wilcoxon, } \\
\text { T-test }\end{array}$ & Age, gender & $\begin{array}{l}\text { Duration of } \\
\text { treatment }\end{array}$ & $\begin{array}{l}\text { Health locus of } \\
\text { control }\end{array}$ & $\begin{array}{l}\text { Composite } \\
\text { score based } \\
\text { on serum } \\
\text { phosphorus, } \\
\text { serum } \\
\text { potassium } \\
\text { and } \\
\text { interdialytic } \\
\text { weight }\end{array}$ & $>6.01 \mathrm{mg} / \mathrm{dl}$ & Not stated \\
\hline $\begin{array}{l}\text { Weed-Collins \& } \\
\text { Hogan, } 1989 \\
\text { U.S.[26] }\end{array}$ & $30 \mathrm{HD}$ & $\begin{array}{l}\text { Not } \\
\text { stated }\end{array}$ & Not stated & $43 \%$ & Correlations & Not assessed & $\begin{array}{l}\text { Not } \\
\text { assessed }\end{array}$ & $\begin{array}{l}\text { Knowledge of } \\
\text { phosphate binders, } \\
\text { susceptibility, } \\
\text { severity, benefits, } \\
\text { barriers* }\end{array}$ & $\begin{array}{l}\text { Serum } \\
\text { phosphorus }\end{array}$ & $>5.5 \mathrm{mg} / \mathrm{dl}$ & $64 \%$ \\
\hline $\begin{array}{l}\text { Wenerowicz et } \\
\text { al, I } 978 \text { U.S.[37] }\end{array}$ & $19 \mathrm{HD}$ & 36 & 7 & $68 \%$ & $\begin{array}{l}\text { Chi-square, } \\
\text { t-test }\end{array}$ & Not assessed & $\begin{array}{l}\text { Not } \\
\text { assessed }\end{array}$ & $\begin{array}{l}\text { Health locus of } \\
\text { control * }\end{array}$ & $\begin{array}{l}\text { Serum } \\
\text { phosphorus }\end{array}$ & $>4.5 \mathrm{mg} / \mathrm{dl}$ & $68 \%$ \\
\hline $\begin{array}{l}\text { Wiebe \& } \\
\text { Christensen, } \\
\text { I } 997 \text { U.S.[48] }\end{array}$ & $70 \mathrm{HD}$ & 56 & $|4|$ & $60 \%$ & $\begin{array}{l}\text { Stepwise, } \\
\text { hierarchical } \\
\text { regression }\end{array}$ & $\begin{array}{l}\text { Age, gender, } \\
\text { education, } \\
\text { marital status }\end{array}$ & $\begin{array}{l}\text { Diabetic } \\
\text { status, time } \\
\text { on dialysis }\end{array}$ & $\begin{array}{l}\text { Conscientiousness, } \\
\text { susceptibility, } \\
\text { severity, benefits, } \\
\text { barriers }\end{array}$ & $\begin{array}{l}\text { Serum } \\
\text { phosphorus }\end{array}$ & $\begin{array}{l}\text { Not } \\
\text { dichotomised }\end{array}$ & Not stated \\
\hline
\end{tabular}

Note: $\mathrm{HD}=$ hemodialysis, $\mathrm{PD}=$ peritoneal dialysis, ESRD $=$ End stage renal disease
$* \mathrm{p}<0.05 * * \mathrm{p}<0.01 *^{* * *} \mathrm{p}<0.001$ relationship with serum phosphorus levels, ${ }^{+} \mathrm{p}<0.05^{++} \mathrm{p}<0.01{ }^{+++} \mathrm{p}<0.001$ relationship with self report adherence, ${ }^{\#} \mathrm{p}<0.05{ }^{\# \#} \mathrm{p}<0.01{ }^{\# \#} \mathrm{p}<0.001$

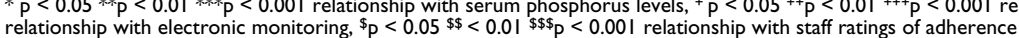

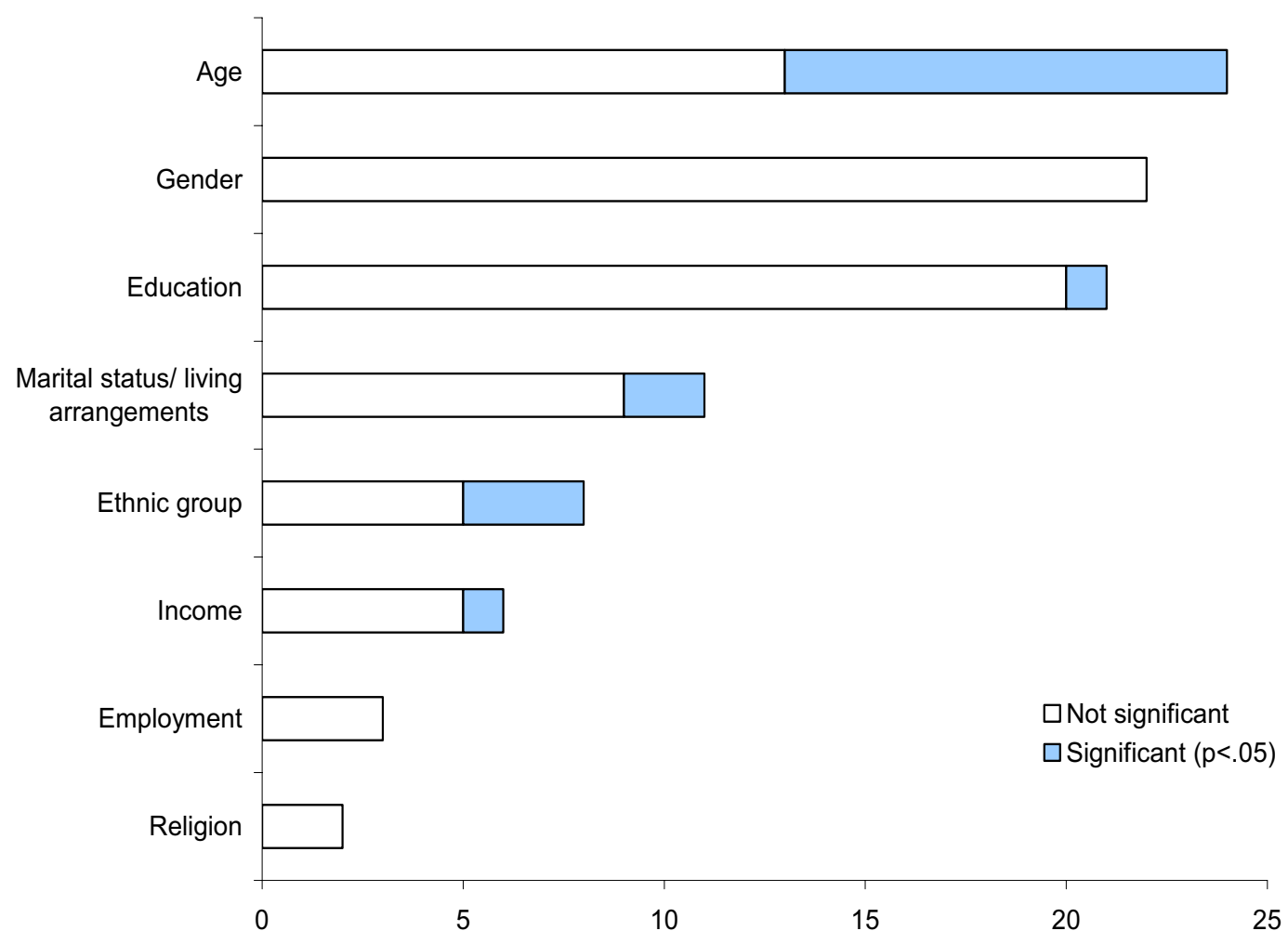

No. studies assessing each possible predictor of nonadherence

Figure I

Demographic predictors of nonadherence to phosphate binding medication assessed by two or more studies. 
studies found significant relationships between demographic factors and adherence to phosphate binding medication, with the exception of those exploring the impact of age on adherence, where 11 of the 24 studies (46\%) exploring this variable found a significant result [12-22]. In these studies, older age was consistently associated with higher levels of adherence. Suggested reasons for this finding are that older people may be more concerned about their mortality and have more structured lives in which to accommodate the demands of the treatment regimen [13], that younger patients may have more difficulty coming to terms with having a chronic condition [13] or simply that younger patients are more willing to report nonadherence than older patients [17].

\section{Clinical variables}

The most frequently assessed clinical predictors of nonadherence were length of time on hemodialysis (22 studies), whether or not the patient was diabetic (9 studies) and the patient's transplant history (i.e. whether or not they had received a kidney transplant in the past; 6 studies). As shown in Figure 2, none of these clinical variables were consistently associated with adherence to phosphate binding medication. Given the large tablet burden associated with phosphate binding medication (patients on average take approximately eight phosphate binding tablets per day [3]), it is surprising that only three studies have explored the effects of regimen complexity on adherence $[3,17,22]$, with two finding significant results. One found a correlation between complexity of the phosphatebinding medicine regimen and serum phosphorus [22] and one found that patients reporting low adherence and those above target phosphorus levels were prescribed significantly more phosphate binder pills per day [3]. Although high tablet burden may be a barrier to adherence for many patients, we cannot assume a causal relationship between high tablet burden and low adherence from these studies. One explanation is that high tablet burden leads to low adherence, but an equally plausible explanation is that low adherence results in poorer phos-

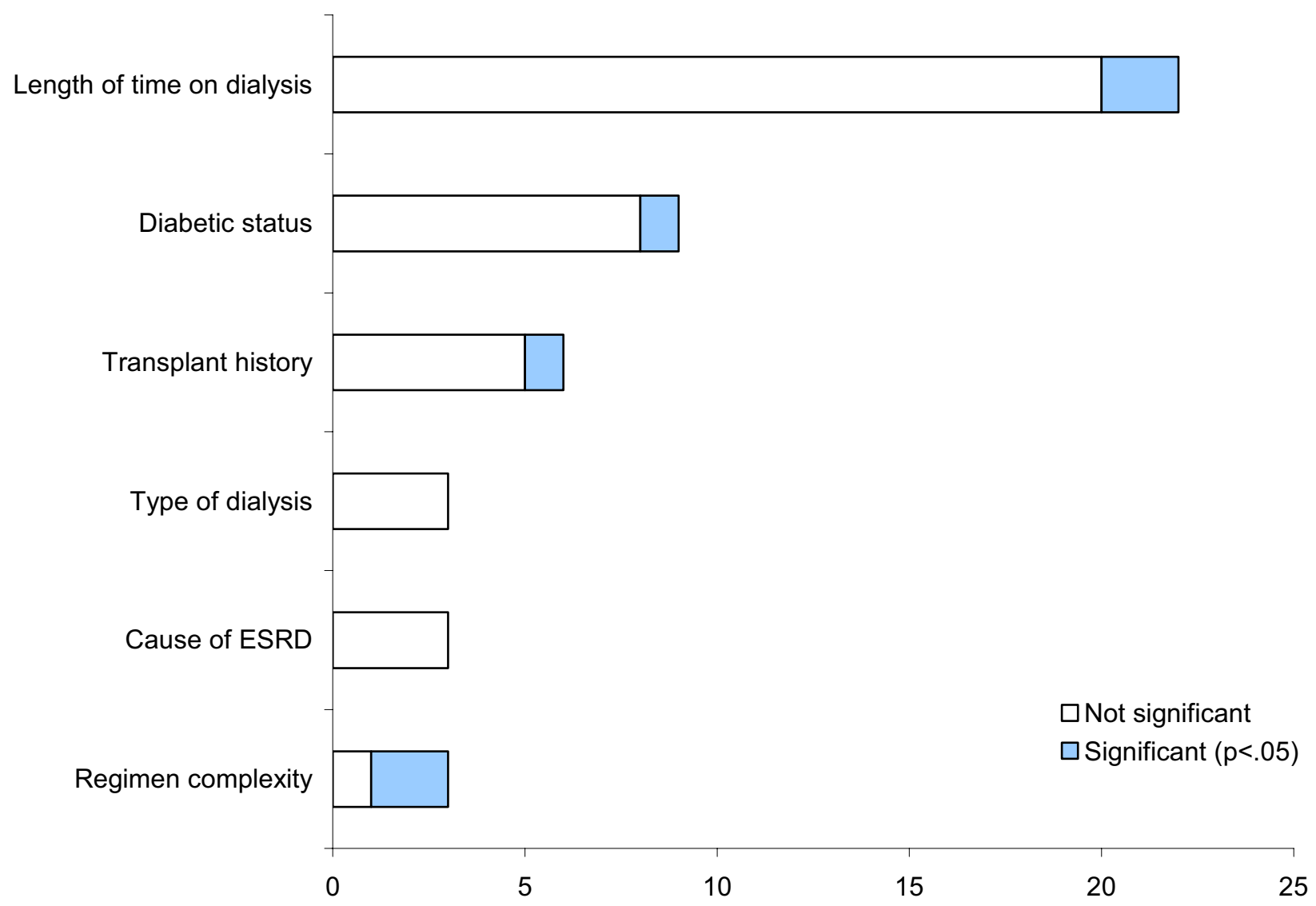

No. studies assessing each possible predictor of nonadherence

\section{Figure 2}

Clinical predictors of nonadherence to phosphate binding medication assessed by two or more studies. 
phate control and an increase in the number of prescribed tablets. A review across other therapeutic areas suggests that prescribed number of doses is inversely related to adherence [23] and this warrants further research in relation to phosphate binding medication. In addition, whilst qualitative and descriptive studies have indicated that the size and taste of the tablets may impact on adherence to phosphate binding medication $[24,25]$, none of the quantitative studies reviewed explored these variables. Further research is needed to determine the role of these tabletrelated factors in predicting nonadherence.

\section{Psychosocial variables}

Whilst psychosocial predictors of nonadherence were the least often assessed, they were more likely to be significantly associated with nonadherence to phosphate binding medication than demographic and clinical variables (see Figure 3).
Six of the nine studies investigating the relationship between health beliefs and adherence to phosphate binding medication reported significant relationships. These beliefs were all related to patients' perceptions of medication (e.g. concerns about potential adverse effects of medication [17], perceived barriers to and benefits of taking medication $[22,26]$, perceptions of self efficacy with regard to taking the medication $[16,27]$ and perceptions of others' expectations regarding adherence [28]). In a recent review of adherence to medication across chronic illnesses, such beliefs were identified as important potentially modifiable predictors of nonaderence that could be addressed within interventions to facilitate adherence [5].

Four of five studies found a relationship between social support and adherence to phosphate binding medication. This included support of friends and family $[13,29,30]$ and of renal staff [22]. In addition, three of five studies exploring associations between family dynamics and adherence reported significant results. Family problems

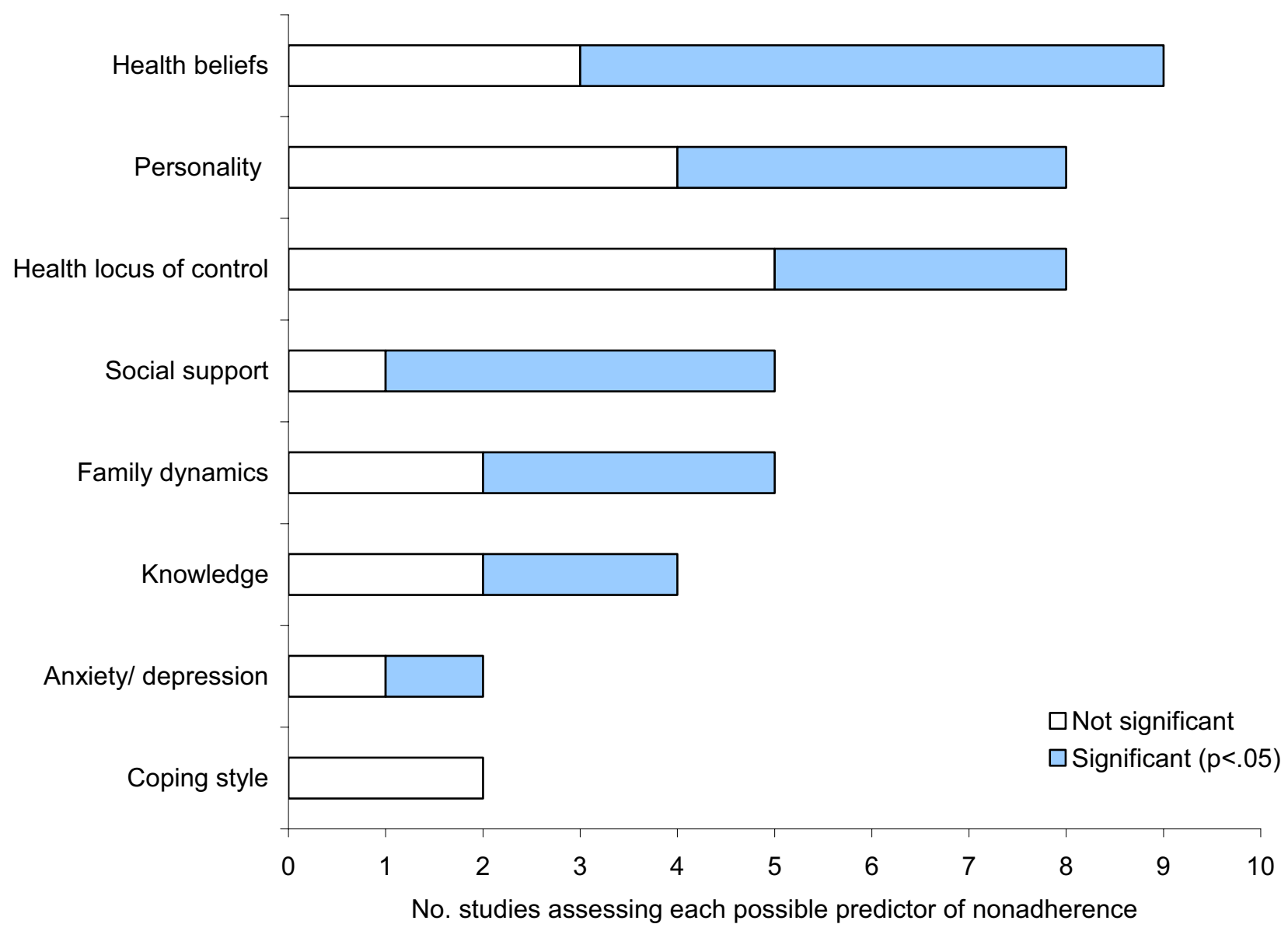

Figure 3

Psychosocial predictors of nonadherence to phosphate binding medication assessed by two or more studies. 
caused by the patient's illness [22], disorganisation and disagreements within the family [30] and lack of clear family structure [31] were associated with low adherence to phosphate binding medication. It is interesting that whilst marital status/living arrangements alone were not often associated with adherence, patients' perceptions of the actual support they received and the quality of their family relationships were more likely to be associated with adherence. This is consistent with findings in the broader social support literature that suggest that it is the quality rather than quantity of social support that is important in predicting mental and physical health outcomes [32].

Four of the eight studies exploring personality as a predictor of adherence to medication found significant results. Personality traits associated with nonadherence included low conscientiousness [14], high cynical hostility [33], and being expedient, venturesome, experimental and lacking self control [34].

Findings of studies looking at knowledge as a predictor of nonadherence were mixed. Two out of four studies found an association between knowledge of the purpose of the regimen and phosphate levels $[20,22]$. However, the other two studies found no relationship between knowledge of treatment instructions and adherence to phosphate binding medication $[26,35]$. Knowledge might be a prerequisite for adherence behaviour but the presence of knowledge alone may not bring about change in behaviour.

\section{Methodological limitations of the studies reviewed}

Several methodological limitations of the studies were noted. These related to the definition and measurement of nonadherence and the study design and sampling.

\section{Adherence assessment methods}

A variety of methods of assessing adherence were utilised in the studies, including tablet counts, electronic monitoring, patient self-report, health care professionals' reports and serum phosphorus levels. Each method has its own limitations, as discussed in a recent review of adherence, compliance and concordance [5]. Serum phosphorus was the most frequently used indicator of phosphate binder adherence (79\% studies). This can be problematic as it reflects not only adherence to phosphate binding medication but also adherence to diet restrictions and dialysis attendance. It has also been suggested that serum phosphorus levels can be affected by 'residual renal function, urine output, co-morbid illnesses, hypercatabolism, nutritional status, hormonal and acid base status, type and intensity of dialytic treatment' [36], highlighting the lack of specificity of this measure. Where studies used more than one method of measuring adherence, rates of nonad- herence and predictors of nonadherence varied depending on the adherence measure used $[3,22,29]$. This makes it very difficult to accurately estimate the levels of nonadherence in the renal dialysis population.

\section{Definitions of nonadherence}

Definitions of nonadherence were inconsistent. Serum phosphorus levels that were considered acceptable ranged from $4.5 \mathrm{mg} / \mathrm{dl} \mathrm{[37]} \mathrm{to} 7.5 \mathrm{mg} / \mathrm{dl} \mathrm{[18]} \mathrm{and} \mathrm{this} \mathrm{was}$ reflected in the reported rates of nonadherence, with the study adopting the highest cut-off reporting the lowest rates of nonadherence $(22 \%,[18])$, and the study adopting the lowest cut-off reporting one of the highest rates of nonadherence $(68 \%,[37])$. Similarly, there was variation in the level of adherence that was considered acceptable in studies using self report measures of nonadherence, with definitions of nonadherence ranging from 'ever missing a dose' [38] to 'missing more than $20 \%$ of doses' [3]. More research is necessary to determine the level of adherence to phosphate binding medication required to prevent negative health outcomes.

\section{Composite measures of adherence}

Eight studies combined adherence to phosphate binding medication with adherence to other parts of the treatment regimen (e.g. attendance at dialysis, adherence to diet and fluid restrictions) for the analysis [28-31,34,39-41]. People may have different levels of adherence for different parts of the treatment regimen and therefore adherence to the individual components should ideally be considered in isolation. Indeed, studies that did assess adherence to different parts of the regimen separately not only reported different levels of adherence to the different aspects of treatment but also found that different factors predicted adherence to different parts of the regimen [12$15,18,21,22,26,27,33,35,42-48]$.

\section{Study design}

Only three studies utilised a prospective design $[14,27,28]$, with the remainder using a cross sectional study design. Whilst cross-sectional studies enable the identification of associations between variables, prospective studies are required to determine causal links between potential predictor variables and adherence.

\section{Sample size}

Many studies had small sample sizes, with a third including less than 50 people and 6 studies $(18 \%)$ reporting sample sizes of 25 or less. Only one study included a power calculation [17] and it is likely that many of the other studies would not have had the power to detect predictors of nonadherence. It is therefore possible that actual predictors of nonadherence remain undetected. Future research should ensure sample sizes are large 
enough for the analysis to identify significant predictors of nonadherence, should they exist.

\section{Health care system bias}

The vast majority of the studies were conducted in the United States of America (79\%). It is possible that the health care system in the United States has unique characteristics that could influence adherence (e.g. prescription charges, private health insurance). It is therefore not possible to generalise the results to all health care systems and there is a need for further research outside of the United States.

\section{Conclusion}

Nonadherence to phosphate binding medication is a serious problem; studies report that $22-74 \%$ patients are nonadherent with their phosphate binding medication, with the variation attributable to differences in the definition and measurement of nonadherence.

Demographic and clinical factors are not consistently associated with nonadherence to phosphate binding medication, with the exception of age (older patients are more likely to be adherent). However issues such as regimen complexity, which are likely to be important determinants of adherence, have not been fully explored and should be considered in future research.

Across studies, psychosocial factors appear to be the most promising predictors of nonadherence, including patients' beliefs about their treatment and their perceived social support. However, limitations in research design and study power create the need for further methodologically sound studies to identify the key beliefs influencing nonadherence to phosphate binders as a basis for the development of interventions to facilitate motivation, informed choice and appropriate adherence.

\section{Competing interests}

The author(s) declare that they have no competing interests.

\section{Authors' contributions}

CK: Literature review, first draft of paper

JC: Literature review, drafting paper, presentation of results

JW: Comments on the drafts

RH: Design of the review, structure of the paper, comments on the draft

\section{Acknowledgements}

This review was supported by an unrestricted educational grant from Shire Pharmaceuticals.

\section{References}

I. United States Renal Data System: Annual Data Report. National Institutes of Health, National Institute of Diabetes and Digestive and Kidney Disease. Bethesda 1998.

2. Block AG, Hulbert-Shearon TE, Levin NW, Port FK: Association of serum phosphorus and Calcium $\times$ Phosphate product with mortality risk in chronic haemodialysis patients: a national study. Am J Kidney Dis 1998, 3 I (4):607-6I7.

3. Tomasello S, Dhupar S, Sherman RA: Phosphate Binders, K/ DOQI Guidelines, and Compliance: The Unfortunate Reality. Dialysis \& Transplantation 2004, 33(5):236-242.

4. World Health Organization: Adherence to Long-term Therapies: Evidence for Action. World Health Organization. 2003.

5. Horne R, Weinman J, Barber N, Elliott RA, Morgan M: Concordance, adherence and compliance in medicine taking: a conceptual map and research priorities. National Co-ordinating Centre for NHS Service Delivery and Organisation NCCSDO, London 2005.

6. Kripalani S, Yao X, Haynes RB: Interventions to enhance medication adherence in chronic medical conditions: a systematic review. Archives of internal medicine 2007, I 67(6):540-550.

7. Hoover $\mathrm{H}$ : Compliance in hemodialysis patients: a review of the literature. J Am Diet Assoc 1989, 89(7):957-959.

8. Hailey B, Moss S: Compliance behaviour in patients undergoing haemodialysis: A review of the literature. Psychology, Health \& Medicine 2000, 5(4):395-406.

9. Richard CJ: Self-care management in adults undergoing hemodialysis. Nephrology Nursing Journal 2006, 33(4):387-396.

10. Morgan L: A decade review: methods to improve adherence to the treatment regimen among hemodialysis patients. Nephrology Nursing Journal: Journal of the American Nephrology Nurses' Association 2000, 27(3):299-304.

II. Ricka R, Vanrenterghem Y, Evers GCM: Adequate self-care of dialysed patients: a review of the literature. Int J Nurs Stud 2002, 39(3):329-339.

12. Bame SI, Petersen N, Wray NP: Variation in hemodialysis patient compliance according to demographic characteristics. Soc Sci Med 1993, 37(8): 1035-1043.

13. Boyer CB, Friend R, Chlouverakis G, Kaloyanides G: Social support and demographic factors influencing compliance of hemodialysis patients. Journal of Applied Social Psychology 1990, 20(22, Pt 2): $1902-1918$.

14. Christensen AJ, Smith TW, Turner CW, Cundick KE: Patient adherence and adjustment in renal dialysis: a person $\times$ treatment interactive approach. Journal of Behavioral Medicine 1994, I 7(6):549-566.

15. Christensen AJ, Smith TW: Personality and patient adherence: correlates of the five-factor model in renal dialysis. J Behav Med 1995, I 8(3):305-313.

16. Christensen AJ, Wiebe JS, Benotsch EG, Lawton WJ: Perceived health competence, health locus of control, and patient adherence in renal dialysis. Cognitive Therapy and Research 1996, 20(4):4||-42|.

17. Horne R, Sumner S, Jubraj B, Weinman J, Frost S: Haemodialysis patients' beliefs about treatment: Implications for adherence to medication and fluid-diet restrictions. International Journal of Pharmacy Practice 2001, 9(3): 169-175.

18. Leggat JE Jr, Orzol SM, Hulbert-Shearon TE, Golper TA, Jones CA, Held PJ, Port FK: Noncompliance in hemodialysis: predictors and survival analysis. Am J Kidney Dis 1998, 32(I): I 39-I45.

19. Schneider B: Multidimensional health locus of control as partial predictor of serum phosphorus in chronic hemodialysis. Psychol Rep 1992, 70(3 Pt 2): I I71-1 I74.

20. Stamatakis MK, Pecora PG, Gunel E: Factors influencing adherence in chronic dialysis patients with hyperphosphatemia. Journal of Renal Nutrition 1997, 7(3): |44-148.

21. Takaki J, Nishi T, Shimoyama H, Inada T, Matsuyama N, Sasaki T, Kumano $\mathrm{H}$, Kuboki T: Associations and interactions of age, sex, and duration of hemodialysis with compliance in uremic patients. Dialysis \& Transplantation 2003, 32(I): I 2-I7.

22. Cummings KM, Becker MH, Kirscht JP, Levin NW: Psychosocial factors affecting adherence to medical regiments in a group of hemodialysis patients. Med Care 1982, 20(6):567-580.

23. Claxton AJ, Cramer J, Pierce C: A systematic review of the associations between dose regimens and medication compliance. Clinical Therapeutics 200I, 23(8): I296-I3 I0. 
24. Karamanidou C, Wienman J, Horne R: A qualitative in-depth study of barriers to adherence to phosphate-binding medication in a small cohort of haemodialysis patients. Poster presentation at the 2007 World Congress of Nephrology, April 2I-25 2007 2007. Rio de Janeiro, Brazil

25. Riley S: Examining patient perceptions of phophate binders. British Journal of Renal Medicine 2007, I 2(I): | 9-2I.

26. Weed-Collins M, Hogan R: Knowledge and health beliefs regarding phosphate-binding medication in predicting compliance. Anna Journal 1989, I6(4):278-282.

27. Eitel P, Friend R, Griffin KW, Wadhwa NK: Cognitive control and consistency in compliance. Psychology \& Health 1998 I3(6):953-973.

28. O'Brien ME: Hemodialysis regimen compliance and social environment: a panel analysis. Nurs Res 1980, 29(4):250-255.

29. Hilbert $G$ : An investigation of the relationship between social support and compliance of hemodialysis patients. ANNA Journal 1985, I 2(2): I33-136.

30. Sherwood RJ: Compliance behavior of hemodialysis patients and the role of the family. Family Systems Medicine 1983, I(2):60-72.

31. Steidl JH, Finkelstein OF, Wexler JP, Feigenbaum H, Kitsen J, Kliger AS, Quinlan DM: Medical condition, adherence to treatment regimens, and family functioning. Their interactions in patients receiving long-term dialysis treatment. Archives of General Psychiatry 1980, 37(9): I025-1027.

32. VanderVoort D: Quality of social support in mental and physical health. Current Psychology 1999, I 8(2):205-222.

33. Christensen AJ, Wiebe JS, Lawton WJ: Cynical hostility, powerful others control expectancies, and patient adherence in hemodialysis. Psychosom Med I997, 59(3):307-3। 2.

34. Schlebusch L, Levin A: Can poor compliance in hemodialysis patients be predicted? Dialysis \& Transplantation 1982 II(7):601-604.

35. Gago C, Gruss E, GÃlzalez S, Marco B, FernÃndez J, Jarriz A, Martinez S, GÃnzalez A, GÃlvez C, Andrea C, et al.: Compliance of haemodialysis patients with prescribed medication. EDTNA/ERCA Journal of Renal Care 2000, 26(4):4-6.

36. Kaveh K, Kimmel PL: Compliance in hemodialysis patients: Multidimensional measurements in search of a gold standard. Am J Kidney Dis 200I, 37:244-266

37. Wenerowicz WJ, Riskind JH, Jenkins PG: Locus of control and degree of compliance in hemodialysis patients. J Dial 1978 2(5-6):495-505.

38. Blanchard R, Berger W, Bailie GR, Eisele G: Knowledge of hemodialysis and CAPD patients about their prescribed medicines. Clinical Nephrology 1990, 34(4): I73-178.

39. Lin CC, Liang CC: The relationship between health locus of control and compliance of hemodialysis patients. Kaohsiung J Med Sci 1997, I3(4):243-254.

40. Tracy HM, Green C, McCleary J: Noncompliance in hemodialysis patients as measured with the MBHI. Psychology \& Health 1987 , I(4):4 I |-423.

4I. Vives T, Pujolar N, Junyent E, Flores I, Cordovilla L, Izquierdo R Adherence to treatment and personality in renal failure. EDTNA/ERCA Journal of Renal Care $1999,25(3): 13-14$

42. Betts DK, Crotty GD: Response to illness and compliance of long-term hemodialysis patients. Anna J 1988, I 5(2):96-99.

43. Christensen AJ, Moran PJ, Lawton WJ, Stallman D, Voigts AL: Monitoring attentional style and medical regimen adherence in hemodialysis patients. Health Psychol 1997, I 6(3):256-262.

44. Christensen AJ, Wiebe JS, Edwards DL, Michels JD, Lawton WJ: Body consciousness, illness-related impairment, and patient adherence in hemodialysis. I Consult Clin Psychol 1996, 64(I): I47-I52.

45. Moran PJ, Christensen AJ, Lawton WJ: Social support and conscientiousness in hemodialysis adherence. Ann Behav Med 1997 I 9(4):333-338.

46. Morduchowicz G, Sulkes J, Aizic S, Gabbay U, Winkler J, Boner G: Compliance in hemodialysis patients: a multivariate regression analysis. Nephron 1993, 64(3):365-368.

47. Reiss D, Gonzalez S, Kramer N: Family process, chronic illness, and death: On the weakness of strong bonds. Archives of Gen eral Psychiatry 1986, 43(8):795-804
48. Wiebe JS, Christensen AJ: Health beliefs, personality, and adherence in hemodialysis patients: an interactional perspective. Ann Behav Med 1997, I9( I):30-35

49. Curtin RB, Svarstad BL, Keller TH: Hemodialysis patients' noncompliance with oral medications. Anna Journal 1999 , 26(3):307-316. discussion 317

\section{Pre-publication history}

The pre-publication history for this paper can be accessed here:

http://www.biomedcentral.com/1471-2369/9/2/prepub

Publish with Biomed Central and every scientist can read your work free of charge

"BioMed Central will be the most significant development for disseminating the results of biomedical research in our lifetime. "

Sir Paul Nurse, Cancer Research UK

Your research papers will be:

- available free of charge to the entire biomedical community

- peer reviewed and published immediately upon acceptance

- cited in PubMed and archived on PubMed Central

- yours - you keep the copyright
BioMedcentral 